\title{
BIRATIONAL SUPERRIGIDITY AND SLOPE STABILITY OF FANO MANIFOLDS
}

\author{
YUJI ODAKA AND TAKUZO OKADA
}

\begin{abstract}
We prove a relation between birational superrigidity of Fano manifold and its slope stability in the sense of Ross-Thomas RT07.
\end{abstract}

Contents

1. Introduction

2. Preliminaries

2.1. Birational (super)rigidity 3

2.2. Seshadri constants 6

2.3. Slope stability 6

3. Exceptional divisors with divisorial center 10

4. Exceptional divisors with higher codimensional center 10

5. A conjecture 13

References 13

\section{INTRODUCTION}

The concept of birational (super)rigidity of Fano manifolds (or of Mori fiber spaces, in general) was introduced to extend the work of Iskovskikh-Manin IM71 for quartic threefolds. The concept emerged in the study of the rationality problem for Fano manifolds by analyzing birational maps between such spaces.

The purpose of this paper is to show a relation between birational (super)rigidity and GIT stability, which is unexpected because of the different nature of their origins. More precisely, in this paper we study slope stability of polarized varieties, which was introduced by RossThomas (cf. [RT07]) as an analogue of Mumford-Takemoto's slope stability of vector bundles. It is also a weaker version of K-stability, which was first formulated by Tian in [Tia97] and later reformulated and

Date: 8th, September, 2011. Revised December, 2012. 
generalized by Donaldson [Don02. (Ross-Thomas [RT07 follow the formulation of [Don02].)

Our main result is the following.

Theorem 1.1. Let $X$ be a birationally superrigid Fano manifold of Fano index 1. If $\left|-K_{X}\right|$ is base point free, then $\left(X, \mathcal{O}_{X}\left(-K_{X}\right)\right)$ is slope stable.

We remark that the assumptions in Theorem 1.1 on the index and the base point freeness of the anticanonical linear system $\left|-K_{X}\right|$ seem to be mild. As far as the authors know, every Fano manifold which has been proved to be birationally superrigid satisfies both assumptions (see section 2.1 for examples of birationally superrigid Fano manifolds).

Actually we prove the following stronger but technical result from which Theorem 1.1 follows.

Theorem 1.2. Let $X$ be a Fano manifold of Picard rank 1 and index 1 with no log maximal singularity (see section 2.1 for the definition). If $\left|-K_{X}\right|$ is base point free, then $\left(X, \mathcal{O}_{X}\left(-K_{X}\right)\right)$ is slope stable.

Recall that the motivation for introducing K-stability is to formulate the following relation with the existence of Kähler metrics, which has been called the Yau-Tian-Donaldson conjecture.

Conjecture 1.3. An arbitrary Fano manifold $X$ with discrete automorphism group admits a Kähler-Einstein metric if and only if $\left(X,-K_{X}\right)$ is K-stable.

The only if direction was finally proved by Stp08 and proofs of the if direction were recently announced by CDS12], [Tia12]. Note that every birationally superrigid Fano manifold has discrete automorphism group since it is not (birationally) ruled. We also refer the readers to [Li13] for a differential geometric counterpart of $K$-semistability as well.

We remark that the following example shows that our result cannot be derived from the main result of OS12.

Example 1.4. Let $X$ be a smooth projective hypersurface of dimension $n$ and degree $n+1$ in $\mathbb{P}^{n+1}$. After the papers [IM71] and [P98], it has been completely proved in $\mathrm{dF} 13$, that $X$ is birationally superrigid for $n \geq 3$. On the other hand, it is known that the alpha invariant $\alpha(X)$ of Tian ([Tia87], cf. also [OS12]) is $\frac{n}{n+1}$ if $X$ contains some generalized Eckardt points (or equivalently, hyperplane sections of cone type) so that strict stability does not directly follow from OS12. We are grateful to Professor Constantin Shramov for pointing this out to us. We refer the readers to OS12 for basics of the alpha invariant and its algebro-geometric version. 
Our proof of Theorem 1.1 is similar to that of [OS12]. Recall that the two fundamental observations in OS12 are that:

- Certain explicit upper bounds for the Seshadri constants imply K-stability of a $\mathbb{Q}-$ Fano variety (see [OS12, Corollary 4.4]).

- Mildness of singularities of pluri-anticanonical divisors gives upper bounds for the Seshadri constants.

We combine these observations to prove Theorem 1.1, which is possible since birational superrigidity asserts certain mildness of singularities of pluri-anticanonical divisors as we will review in subsection 2.4.

In the next section, we give some basic definitions and review the background. In section 3, we prove stability along divisors and in section 4, we prove stability along higher codimensional loci. The last section proposes a more general conjecture about stability of Fano manifolds.

We work over the field of complex numbers $\mathbb{C}$ throughout this paper.

Acknowledgments. We are grateful to Professors Shigefumi Mori, Constantin Shramov and Alexander Pukhlikov for helpful comments. We would like to thank Doctors Jesus Martinez-Garcia and Julius Ross for their careful reading of the draft versions and Professor Ivan Cheltsov for his interest in this work. We also would like to thank the referee for her/his efforts to point out many errors. The first author is partially supported by the Grant-in-Aid for Scientific Research (KAKENHI No. 21-3748) and the Grant-in-Aid for JSPS fellows (DC1). The second author is partially supported by the Grand-in-Aid for Scientific Research (KAKENHI No. 23-2053) and the Grand-in-Aid for JSPS fellows (PD).

\section{Preliminaries}

2.1. Birational (super)rigidity. In this subsection, we recall the definition of birational (super)rigidity and its basic properties. We refer the readers to [P07] for an introduction to this subject.

Definition 2.1. Let $\pi: V \rightarrow S$ be a projective surjective morphism between normal projective varieties with connected fibers. We say that $\pi: V \rightarrow S$ is a Mori fiber space if

(i) $V$ is $\mathbb{Q}$-factorial and has at most terminal singularities,

(ii) $-K_{V}$ is $\pi$-ample,

(iii) $\operatorname{dim} S<\operatorname{dim} V$, and

(iv) the relative Picard number $\rho(V / S)$ is 1 .

Throughout this subsection, let $X$ be a $\mathbb{Q}$-factorial $\mathbb{Q}$-Fano variety with Picard number 1 and with at most terminal singularities. Note 
that $X$, together with the structure morphism (to a point), can be seen as a Mori fiber space. Although birational (super)rigidity can be defined for any Mori fiber space, we only give the definition for $\mathbb{Q}$-Fano varieties with Picard number one.

Definition 2.2. We say that $X$ is birationally rigid if for any birational map $\varphi: X \rightarrow X^{\prime}$ to a Mori fiber space there is a birational selfmap $\tau: X \rightarrow X$ such that $\varphi \circ \tau: X \rightarrow X^{\prime}$ can be extended to an isomorphism. We say that $X$ is birationally superrigid if in the above definition of birational rigidity one can always take $\tau=\mathrm{id}_{X}$.

It is easy to see that $X$ is birationally superrigid if and only if $X$ is birationally rigid and $\operatorname{Bir}(X)=\operatorname{Aut}(X)$. Let $\mathcal{H}$ be a movable linear system on $X$, that is, a linear system without fixed components. We define $\mu=\mu(X, \mathcal{H})$ to be the rational number for which $\mu K_{X}+\mathcal{H} \equiv 0$, where $\equiv$ denotes the numerical equivalence. Let $\lambda$ be a nonnegative rational number. We say that a pair $(X, \lambda \mathcal{H})$ is terminal (resp. canonical, resp. log canonical) if every rational number $a(X, \lambda \mathcal{H}, E)$ determined by

$$
K_{V}+\lambda f_{*}^{-1} \mathcal{H}=f^{*}\left(K_{X}+\lambda \mathcal{H}\right)+\sum a(X, \lambda \mathcal{H}, E) E,
$$

is positive (resp. non-negative, resp. $\geq-1$ ) for every birational morphism $f: V \rightarrow X$, where $E$ runs over the $f$-exceptional prime divisors.

The canonical threshold (resp. log canonical threshold) of the pair $(X, \mathcal{H})$ is defined to be the number

$$
\operatorname{ct}(X, \mathcal{H}):=\sup \left\{\lambda \in \mathbb{Q}_{>0} \mid(X, \lambda \mathcal{H}) \text { is canonical }\right\}
$$

(resp. $\operatorname{lct}(X, \mathcal{H}):=\sup \left\{\lambda \in \mathbb{Q}_{>0} \mid(X, \lambda \mathcal{H})\right.$ is $\log$ canonical $\left.\}\right)$.

Definition 2.3. We say that $X$ has a maximal singularity (resp. log maximal singularity) if there is a movable linear system $\mathcal{H}$ on $X$ such that $\left(X, \frac{1}{\mu} \mathcal{H}\right)$ is not canonical (resp. not log canonical) for $\mu=\mu(X, \mathcal{H})$. We say that $X$ is maximal singularity free (resp. log maximal singularity free) if $X$ does not have a maximal singularity (resp. log maximal singularity).

The Noether-Fano-Iskovskikh inequality [Co95, Theorem 4.2] shows that if $X$ is maximal singularity free then it is birationally superrigid. We use (log) maximal singularity freeness to prove stability in the following sections. The following result shows that maximal singularity freeness characterizes birational superrigidity.

Theorem 2.4 (Cheltsov-Shramov [CS08, Theorem 1.26]). $X$ is birationally superrigid if and only if it is maximal singularity free. 
We now review some examples. The following are Fano threefolds which have been proved to be birationally superrigid.

- A smooth quartic threefold [IM71].

- A sextic double solid, that is, a double cover $X \rightarrow \mathbb{P}^{3}$ ramified along a surface $S \subset \mathbb{P}^{3}$ of degree 6 [I79].

The following are higher-dimensional examples.

- A smooth hypersurface $X_{n+1} \subset \mathbb{P}^{n+1}$ of degree $n+1$, with $n \geq 4$ [AF13, $\mathrm{P} 98$.

- A general complete intersection $X_{d_{1}, \ldots, d_{k}} \subset \mathbb{P}^{n+k}$ of hypersurfaces of degree $d_{i}$ with $d_{i} \geq 2, \sum_{i=1}^{k} d_{i}=n+k>3 k$ and $n \geq 4$ P01.

- A smooth complete intersection $X_{2,4} \subset \mathbb{P}^{6}$ of a quadric and a quartic which does not contain a plane [C03].

- A double cover $X \rightarrow \mathbb{P}^{n}$ ramified along a hypersurface $F \subset \mathbb{P}^{n}$ of degree $2 n$, with $n \geq 4$ [P97].

- A cyclic triple cover $X \rightarrow \mathbb{P}^{2 n}$ ramified along a hypersurface $F \subset \mathbb{P}^{2 n}$ of degree $3 n$ with $n \geq 2$ C04].

- A general cyclic cover $X \rightarrow V \subset \mathbb{P}^{n}$ of degree $d \geq 2$ ramified along a smooth divisor $R \subset V$ such that $V$ is a hypersurface of degree $m \geq 2, m+(d-1) k=n$, where $k$ is a positive integer such that $\mathcal{O}_{V}(R) \cong \mathcal{O}_{V}(d k), n \geq 5$ and either $d=2$ or $n \geq 6$ [P00], [P09].

- A general weighted complete intersection in a weighted projective space

$$
\mathbb{P}\left(1^{l+1}, a_{1}, \ldots, a_{m}\right)=\operatorname{Proj}\left(\mathbb{C}\left[x_{0}, \ldots, x_{l}, y_{1}, \ldots, y_{m}\right]\right)
$$

of $m+k$ hypersurfaces $y_{i}^{2}=g_{i}\left(x_{0}, \ldots, x_{l}\right), i=1, \ldots, m$, and $f_{j}\left(x_{0}, \ldots, x_{l}\right)=0, j=1, \ldots, k$, of degree $2 l_{i}$ and $d_{j}$, respectively, such that

$$
\sum_{i=1}^{m} a_{i}+\sum_{i=1}^{k} d_{i}=l, l>3 k \text { and } l-k \geq 4 .
$$

This is an iterated double cover of general complete intersection in projective space [P03].

For each of the above examples of Fano varieties, we assume the variety is smooth. In some examples we can allow some mild singularities or drop the generality assumptions while keeping the property of birational superrigidity. We refer the readers to [C05] for a detailed account of this subject. We see that every birationally superrigid Fano manifold in the above examples has index 1 and has a base point free anticanonical divisor. 
2.2. Seshadri constants. Let $I \subset \mathcal{O}_{X}$ be a coherent ideal on $X$. The Seshadri constant of $I$ with respect to an ample $\mathbb{Q}$-line bundle $L$ is defined by

$$
\operatorname{Sesh}(I ;(X, L)):=\sup \left\{c \in \mathbb{Q}_{>0} \mid \pi^{*} L(-c E) \text { is ample }\right\},
$$

where $\pi: B l_{I}(X) \rightarrow X$ is the blow up of $X$ along $I$ and $E$ is the associated exceptional Cartier divisor, i.e., $\mathcal{O}(-E)=\pi^{-1} I$. This invariant plays a key role in this paper as in [HKLP11], OS12], [F11] and [F13].

2.3. Slope stability. Consult [Don02, Chapter 2, especially 2.3], [RT07, especially Section 3] or [Od13a, Definition 2.4] for more general background. We remark that our formulation below is formally different from the original presentation by Ross-Thomas [RT07, but they are equivalent, as proved in [RT07, Theorem 4.18]. See below for a more detailed explanation. Let $(X, L)$ be an $n$-dimensional polarized variety.

A test configuration (resp. a semi-test configuration) for $(X, L)$ is a polarized scheme $(\mathcal{X}, \mathcal{M})$ with a $\mathbb{G}_{m}$-action on $(\mathcal{X}, \mathcal{M})$ and a proper flat morphism $\Pi: \mathcal{X} \rightarrow \mathbb{A}^{1}$ such that

(i) $\Pi$ is $\mathbb{G}_{m}$-equivariant for the multiplicative action of $\mathbb{G}_{m}$ on $\mathbb{A}^{1}$,

(ii) $\mathcal{M}$ is relatively ample (resp. relatively semi-ample), and

(iii) $\left.(\mathcal{X}, \mathcal{M})\right|_{\Pi^{-1}\left(\mathbb{A}^{1} \backslash\{0\}\right)}$ is $\mathbb{G}_{m}$-equivariantly isomorphic to $\left(X, L^{\otimes r}\right) \times\left(\mathbb{A}^{1} \backslash\{0\}\right)$ for some positive integer $r$.

If $\mathcal{X} \simeq X \times \mathbb{A}^{1},(\mathcal{X}, \mathcal{M})$ is called a product test configuration. Moreover, if $\mathbb{G}_{m}$ acts trivially, we call it a trivial test configuration.

Slope stability deals with certain special semi-test configurations, called deformation to the normal cone. The definition is as follows. Take a coherent ideal $I \subset \mathcal{O}_{X}$ and set $\mathcal{J}:=I+(t) \subset \mathcal{O}_{X \times \mathbb{A}^{1}}$. Then, for $r \in \mathbb{Z}_{>0}$ with $r>($ resp. $\geq) \frac{1}{\operatorname{Sesh}(I ;(X, L))}$, we set $f: \mathcal{B}:=B l_{\mathcal{J}}(X \times$ $\left.\mathbb{A}^{1}\right) \rightarrow X \times \mathbb{A}^{1}, \mathcal{L}:=f^{*}\left(L \times \mathbb{A}^{1}\right)$ and $\mathcal{O}_{\mathcal{B}}(-E)=f^{-1} \mathcal{J}$ for an effective exceptional Cartier divisor $E$. We note that $\left(\mathcal{B}, \mathcal{L}^{\otimes r}(-E)\right)$ naturally becomes a test configuration (resp. semi-test configuration, if $\mathcal{L}^{\otimes r}(-E)$ is semiample). We call such a test configuration a deformation to the normal cone as in [RT07].

First, let us recall the general definition of the Donaldson-Futaki invariant of a test configuration $(\mathcal{X}, \mathcal{M})$. For $k$ large, let $P(k):=$ $\operatorname{dim} H^{0}\left(X, L^{\otimes k}\right)$, which is a polynomial in $k$ of degree $n$ due to the Riemann-Roch theorem. Since the $\mathbb{G}_{m}$-action preserves the central fibre $\mathcal{X}_{0}$ of $\mathcal{X}, \mathbb{G}_{m}$ acts also on $H^{0}\left(\mathcal{X}_{0},\left.\mathcal{M}^{\otimes s}\right|_{\mathcal{X}_{0}}\right)$, where $s \in \mathbb{Z}_{>0}$. Let $w(r s)$ be the weight of the induced action on the highest exterior power of $H^{0}\left(\mathcal{X}_{0},\left.\mathcal{M}^{\otimes s}\right|_{\mathcal{X}_{0}}\right)$, which is a polynomial in $s$ of degree $n+1$ due to Mumford's droll Lemma (cf. [Mum77, Lemma 2.14] and Od13a, 
Lemma 3.3]) and the Riemann-Roch theorem. Recall that, since an arbitrary $\mathbb{G}_{m}$-action on a finite-dimensional vector space is diagonalizable, we can define the weights as the exponents of the eigenvalues of the action. The total weight of such an action is defined as the sum of all those weights. Now take the $r P(r)$-th power of the action of $\mathbb{G}_{m}$ on $\left.\left(\Pi_{*} \mathcal{M}\right)\right|_{\{0\}}$ and SL-normalize it. Then the corresponding normalized weight on $\left.\left(\Pi_{*} \mathcal{M}^{\otimes K}\right)\right|_{\{0\}}$ is

$$
\tilde{w}_{r, K r}:=w(k) r P(r)-w(r) k P(k),
$$

where $k:=K r$. $\tilde{w}_{r, K r}$ is a polynomial of form $\sum_{i=0}^{n+1} e_{i}(r) k^{i}$ of degree $n+1$ in $k$ for $k \gg 0$, with coefficients which are also polynomials of degree $\leq n+1$ in $r$ for $r \gg 0: e_{i}(r)=\sum_{j=0}^{n+1} e_{i, j} r^{j}$ for $r \gg 0$. Since the weight is normalized, $e_{n+1, n+1}=0$. The coefficient $e_{n+1, n}$ is called the Donaldson-Futaki invariant of the test configuration, which we denote by $\operatorname{DF}(\mathcal{X}, \mathcal{M})$. For an arbitrary semi test configuration $(\mathcal{X}, \mathcal{M})$ of order $r$, we can define the Donaldson-Futaki invariant by setting $w(K r)$ as the total weight of the induced action on $H^{0}\left(\mathcal{X}, \mathcal{M}^{\otimes K}\right) / t H^{0}\left(\mathcal{X}, \mathcal{M}^{\otimes K}\right)$ (cf. [RT07]). Now we can define the stability notions we are concerned with.

Definition 2.5 ([RT07]). We say that $(X, L)$ is slope stable (resp. slope semistable) if and only if the Donaldson-Futaki invariant is positive (resp., non-negative) for any non-trivial deformation to the normal cone.

Note that the above definition is a priori different from the one given in [RT07]. Nevertheless, they are proved to be equivalent in [RT07, proof of Theorem 4.18]. (Of course, there are no essential differences but we will follow our formulation 2.5 just because we are more accustomed to treat the stability in this way.)

The following definition follows the formulation of Donaldson [Don02, slightly modified 1 in [LX11] (cf. also Od13b]).

Definition 2.6. Suppose for simplicity that $X$ is normal. We say that $(X, L)$ is $K$-stable (resp. K-semistable) if and only if the DonaldsonFutaki invariant is positive (resp., non-negative) for any non-trivial normal test configuration.

1 This modification is due to a technical issue. For any polarized variety we can find a class of test configurations which are $\mathbb{G}_{m}$-equivariantly isomorphic to trivial test configuration, away from closed subschemes of codimension at least two. Note that those normalizations are the trivial test configurations. The DonaldsonFutaki invariant of the normalization of these "pathological" test configurations automatically vanish. The modification of the definition excluded those class by considering only normal test configurations. 
We end this subsection with a small remark on an extension of the above framework. If we take a test configuration (resp. semitest configuration) $(\mathcal{X}, \mathcal{M})$, we can think of a new test configuration (resp. semi-test configuration) $\left(\mathcal{X}, \mathcal{M}^{\otimes a}\right)$ with $a \in \mathbb{Z}_{>0}$. From the definition of Donaldson-Futaki invariant above, we easily see that $\operatorname{DF}\left(\left(\mathcal{X}, \mathcal{M}^{\otimes a}\right)\right)=a^{n} \operatorname{DF}((\mathcal{X}, \mathcal{M}))$. Therefore, we can define K-stability (also K-polystability and K-semistability) of a pair $(X, L)$ of a projective scheme $X$ and an ample $\mathbb{Q}$-line bundle $L$.

A key ingredient for our study is the following formula, proved in Od13a, to estimate the Donaldson-Futaki invariant for a deformation to the normal cone $(\mathcal{B}, \mathcal{L}(-E))$ derived from the (flag) ideal $\mathcal{J}:=$ $I+(t) \subset \mathcal{O}_{X \times \mathbb{A}^{1}}$.

In the following, let $X$ be a Fano $n$-fold, $I \subset \mathcal{O}_{X}$ an ideal and $L=\mathcal{O}_{X}\left(-r K_{X}\right)$ for some $r \in \mathbb{Z}_{>0}$ with $r \geq 1 / \operatorname{Sesh}\left(I ;\left(X,-K_{X}\right)\right)$. We assume that $\mathcal{L}(-E)$ is semi-ample so that the corresponding polarized scheme $(\mathcal{B}, \mathcal{L}(-E))$ is a semi-test configuration (a deformation to the normal cone).

Theorem 2.7 (cf. Od13a, Theorem 3.2]). Assume that $X$ is a Fano nfold and let $\left(\overline{\mathcal{B}}:=B l_{\mathcal{J}}\left(X \times \mathbb{P}^{1}\right), \overline{\mathcal{L}}(-E)\right)$ be the natural compactification of $(\mathcal{B}, \mathcal{L}(-E))$. Let $p_{i}(i=1,2)$ be the projection from $X \times \mathbb{P}^{1}$ to the $i$-th factor. Suppose that $\mathcal{L}(-E)$ on $\mathcal{B}$ is semi-ample. We denote the normalization of $\mathcal{B}$ by $\tilde{\mathcal{B}}$ and use the same symbols for the pullbacks to $\tilde{\mathcal{B}}$ of the original polarization $\overline{\mathcal{L}}$ and the Cartier divisor $E$. Then, the corresponding Donaldson-Futaki invariant has the following lower bound:

$$
\begin{aligned}
& 2 n !(n+1) ! \mathrm{DF}(\mathcal{B}, \mathcal{L}(-E)) \\
& \quad \geq-\left((\overline{\mathcal{L}}-E)^{n} \cdot \overline{\mathcal{L}}+n E\right)+(n+1) r\left((\overline{\mathcal{L}}-E)^{n} \cdot K_{\tilde{\mathcal{B}} / X \times \mathbb{A}^{1}}\right) \\
& \quad=-\left((\overline{\mathcal{L}}-E)^{n} \cdot \overline{\mathcal{L}}\right)+\left((\overline{\mathcal{L}}-E)^{n} \cdot\left((n+1) r K_{\tilde{\mathcal{B}} / X \times \mathbb{A}^{1}}-n E\right)\right) .
\end{aligned}
$$

The right hand side is just the Donaldson-Futaki invariant of $(\tilde{\mathcal{B}}, \mathcal{L}(-E))$ by Od13a, Theorem3.2] so that the inequality follows from [RT07, Proposition 5.1, Remark 5.2]. See [RT07] and [Od13a for more general statements.

We note that $\frac{1}{r} \leq \operatorname{Sesh}\left(I,\left(X,-K_{X}\right)\right)$ follows from the assumption of the semiampleness of $\mathcal{L}(-E)$ on $\mathcal{B}$.

Proposition 2.8 ([OS12, Proposition4.3]). The inequality

$$
-\left((\overline{\mathcal{L}}-E)^{n} \cdot \overline{\mathcal{L}}\right) \geq 0
$$

holds for any ideal $I \subset \mathcal{O}_{X}$ and equality holds if and only if $\operatorname{dim}\left(\operatorname{Supp}\left(\mathcal{O}_{X} / I\right)\right)=0$. 
Combining Theorem 2.7 and Proposition 2.8, we have the following criterion for the positivity of the Donaldson-Futaki invariant.

Lemma 2.9. Let $X$ be a Fano manifold and let $r \in \mathbb{Z}_{>0}, L=$ $\mathcal{O}_{X}\left(-r K_{X}\right), I \subset \mathcal{O}_{X}$ be as above. Assume that the Weil divisor

$$
(n+1) K_{\tilde{\mathcal{B}} / X \times \mathbb{A}^{1}}-n \operatorname{Sesh}\left(I ;\left(X,-K_{X}\right)\right) E
$$

is effective. Assume moreover it is nonzero if $\left.\operatorname{dim} \operatorname{Supp}\left(\mathcal{O}_{X} / I\right)\right)=0$. Then $\operatorname{DF}(\mathcal{B}, \mathcal{L}(-E))>0$.

Following the convention of OS12], we define $a_{i}$ and $c_{i}$ as

$$
\begin{aligned}
K_{\tilde{\mathcal{B}} / X \times \mathbb{A}^{1}} & =\sum_{i} a_{i} E_{i}, \\
\tilde{\Pi}^{-1} \mathcal{J} & =\mathcal{O}_{\tilde{\mathcal{B}}}\left(-\sum_{i} c_{i} E_{i}\right),
\end{aligned}
$$

where $E_{i}$ 's are exceptional prime divisors. Denote $\sum c_{i} E_{i}$ by $E$. The assumption of Lemma 2.9 can be reformulated as the effectivity of $K_{\tilde{\mathcal{B}} / X \times \mathbb{A}^{1}}-n \operatorname{Sesh}\left(I ;\left(X,-K_{X}\right)\right) E$ i.e.,

$$
\operatorname{Sesh}\left(I ;\left(X,-K_{X}\right)\right) \leq\left(\frac{n+1}{n}\right) \min _{i}\left\{\frac{a_{i}}{c_{i}}\right\} .
$$

Moreover, if $\operatorname{dim} \operatorname{Supp}\left(\mathcal{O}_{X \times \mathbb{A}^{1}} / \mathcal{J}\right)=0$, the divisor needs to be nonzero and effective, i.e.,

$$
\operatorname{Sesh}\left(I ;\left(X,-K_{X}\right)\right)<\frac{(n+1) a_{i}}{n c_{i}}
$$

for some $i$.

We shall prove the main theorems in Sections 3 and 4 by verifying assumptions of Lemma 2.9. Before going to the proof, we deform $I$ slightly as follows under the assumption that $\left|-K_{X}\right|$ is base point free. We write $I=\mathcal{O}_{X}(-F) I^{\prime}$ where $F \in\left|-m K_{X}\right|$ for some $m \in \mathbb{Z}_{>0}$, and $I^{\prime}$ is an ideal with $\operatorname{codim}\left(\operatorname{Supp}\left(\mathcal{O} / I^{\prime}\right)\right) \geq 2$. Then, we replace $F$ with a general member of $\left|-m K_{X}\right|$ so that we obtain new $I$ with the following property.

Condition 2.10. For the blow up $\Pi: \mathcal{B} \rightarrow X \times \mathbb{A}^{1}$ along $\mathcal{J}:=I+(t)$, if $\operatorname{codim}\left(\Pi\left(E_{i}\right) \subset X \times\{0\}\right) \geq 2$ for a $\Pi$-exceptional divisor $E_{i}$, then $\Pi\left(E_{i}\right) \nsubseteq \operatorname{Supp}(F)$.

This replacement is possible since $\left|-m K_{X}\right|$ is base point free by our assumption. Note that the Seshadri constant $\operatorname{Sesh}\left(I ;\left(X,-K_{X}\right)\right)$ does not change by this deformation. Thus, we can take the corresponding semi-test configuration $\mathcal{B}:=B l_{I+(t)}\left(X \times \mathbb{A}^{1}\right)$ for that perturbed $I$. It 
has the same value for the Donaldson-Futaki invariant, which follows from the description via slope ([RT07]).

Thus, we can assume Condition 2.10 for $I$ from now on, in order to estimate the Donaldson-Futaki invariants.

\section{ExCEPTIONAL DiVisors With DIVISORIAL CENTER}

In this section, under the assumptions on $X$ as in Theorem 1.2, we will prove the inequality (2) for $E_{i}$ in the case where $\Pi\left(E_{i}\right)$ is a divisor in $X \times\{0\}$. Let us recall that we denoted $\mathcal{J}=I+(t)$. As in the previous section, we write $I=I^{\prime} \mathcal{O}_{X}(-F)$ for a coherent ideal $I^{\prime} \subset \mathcal{O}_{X}$ satisfying $\operatorname{codim}\left(\operatorname{Supp}\left(\mathcal{O}_{X} / I^{\prime}\right)\right) \geq 2$ and a divisor $F=\sum_{j} d_{j} D_{j}$, where each $D_{j}$ is a prime divisor. Let $D_{i}$ be the component of $F$ such that $D_{i} \times\{0\}$ is the center $\Pi\left(E_{i}\right)$ of $E_{i}$.

First, we claim that the inequality

$$
\operatorname{Sesh}\left(I,\left(X,-K_{X}\right)\right) \leq \frac{1}{d_{i}}
$$

holds. Let $\pi: X^{\prime} \rightarrow X$ be the blow up of $X$ along $I^{\prime}$ and write $\pi^{-1} I^{\prime}=$ $\mathcal{O}_{X^{\prime}}\left(-E^{\prime}\right)$. Then we have $\pi^{-1} I=\mathcal{O}_{X^{\prime}}\left(-E^{\prime}-F^{\prime}\right)$, where $F^{\prime}=\pi^{*} F$. Since $-K_{X}$ is a positive generator of $\operatorname{Pic}(X)$, we see that $F=\sum_{j} d_{j} D_{j}$ is linearly equivalent to $r\left(-K_{X}\right)$ for some positive integer $r$. Note that $r \geq d_{i}$ since $F \geq d_{i} D_{i}$. If the divisor

$$
\pi^{*}\left(-K_{X}\right)-c\left(E^{\prime}+F^{\prime}\right) \sim(1-c r) \pi^{*}\left(-K_{X}\right)-c E^{\prime}
$$

is ample for some $c \in \mathbb{Q}_{>0}$ then $1-c r>0$. This shows that $c<$ $1 / r \leq 1 / d_{i}$, hence we get the desired inequality, from the definition of Seshadri constant.

Second we have

$$
\frac{1}{d_{i}} \leq \frac{a_{i}}{c_{i}}<\frac{(n+1) a_{i}}{n c_{i}}
$$

since the pair $\left(X \times \mathbb{A}^{1}, D_{i} \times \mathbb{A}^{1}\right)$ is canonical around the generic point of $D_{i} \times\{0\}$ and the discrepancy of $\left(X \times \mathbb{A}^{1}, D_{i} \times \mathbb{A}^{1}\right)$ at $E_{i}$ is $a_{i}-c_{i} / d_{i}$. As a conclusion, we get the desired inequality (2) for $E_{i}$ with $\operatorname{dim}\left(\Pi\left(E_{i}\right)\right)=$ $n-1$. We note that the condition $\rho=\operatorname{index}(X)=1$ is sufficient for the arguments in this section.

\section{EXCEPTIONAL DIVISORS WITH HIGHER CODIMENSIONAL CENTER}

In this section, under the same assumptions on $X$, we will prove the inequality (2) for $E_{i}$ in the case where $\operatorname{codim}\left(\Pi\left(E_{i}\right) \subset X \times\{0\}\right) \geq 2$. Recall that we have Condition 2.10 for $I$.

We first note that $\operatorname{Sesh}\left(I,\left(X,-K_{X}\right)\right) \leq \operatorname{Sesh}\left(I^{\prime},\left(X,-K_{X}\right)\right)$ since $\operatorname{Supp}\left(\mathcal{O}_{X} / I^{\prime}\right)$ is not contained in $\operatorname{Supp}(F)$. Take a positive rational 
number $c<\operatorname{Sesh}\left(I,\left(X,-K_{X}\right)\right) \leq \operatorname{Sesh}\left(I^{\prime},\left(X,-K_{X}\right)\right)$. For a sufficiently divisible positive interger $l$, set the linear system $\Sigma_{I^{\prime}, l}^{(c)} \subset\left|-l K_{X}\right|$ which corresponds to $H^{0}\left(X, I^{\prime c l} \mathcal{O}_{X}\left(-l K_{X}\right)\right) \subset H^{0}\left(X, \mathcal{O}_{X}\left(-l K_{X}\right)\right)$. The linear system $\Sigma_{I^{\prime}, l}^{(c)}$ is movable since codim $\operatorname{Supp}\left(\mathcal{O}_{X} / I^{\prime}\right) \geq 2$ and $c<\operatorname{Sesh}\left(I^{\prime},\left(X,-K_{X}\right)\right)$. By log maximal singularity freeness, the pair $\left(X, \frac{1}{l} \Sigma_{I^{\prime}, l}^{(c)}\right)$ is $\log$ canonical, hence the pair $\left(X, \frac{1}{l} D\right)$ is $\log$ canonical for a general member $D$ of $\Sigma_{I^{\prime}, l}^{(c)}$. By the inversion of adjunction on log canonicity $\left[\mathrm{K}+92,17.7\right.$ Theorem], the pair $\left(X \times \mathbb{A}^{1}, \frac{1}{l}\left(D \times \mathbb{A}^{1}\right)+X \times\{0\}\right)$ is $\log$ canonial near $X \times\{0\}$. The discrepancy of the latter pair at $E_{i}$ is $a_{i}-\frac{1}{l} l c c_{i}^{\prime}-b_{i}=a_{i}-c c_{i}^{\prime}-b_{i}$, where $c_{i}^{\prime}:=\operatorname{val}_{E_{i}}\left(I^{\prime}\right)$, the algebraic valuation of $I^{\prime}$ with respect to $E_{i}$, and $b_{i}$ is defined by $\tilde{\Pi}^{*}(X \times\{0\})=\tilde{\Pi}_{*}^{-1}(X \times\{0\})+\sum_{i} b_{i} E_{i}$. Note that $b_{i} \geq 1$ since $X \times\{0\}$ is a Cartier divisor and the center of $E_{i}$ on $X \times \mathbb{A}^{1}$ is contained in $X \times\{0\}$. The log canonicity implies $a_{i}-c c_{i}^{\prime}-b_{i} \geq-1$, which together with $b_{i} \geq 1$ shows the inequality $c \leq a_{i} / c_{i}^{\prime}$. Since this holds for any such $c$, we have

$$
\operatorname{Sesh}\left(I,\left(X,-K_{X}\right)\right) \leq \min _{E_{i} \subset \operatorname{Exc}(\Pi)}\left\{\frac{a_{i}}{c_{i}^{\prime}}\right\},
$$

where $\operatorname{Exc}(\Pi)$ is the exceptional locus of $\Pi$. If $F \equiv-m K_{X}$ with $m \in \mathbb{Z}_{>0}$, the inequality (3) is equivalent to the inequality

$$
\operatorname{Sesh}\left(I,\left(X,-K_{X}\right)\right) \leq \frac{\min \left\{\frac{a_{i}}{c_{i}^{\prime}}\right\}}{1+m \cdot \min \left\{\frac{a_{i}}{c_{i}^{\prime}}\right\}} .
$$

By Condition 2.10, we have $c_{i}=\operatorname{val}_{E_{i}}(\mathcal{J}) \leq \operatorname{val}_{E_{i}}(I)=\operatorname{val}_{E_{i}}\left(I^{\prime}\right)=: c_{i}^{\prime}$.

Summing up,

$$
\operatorname{Sesh}\left(I,\left(X,-K_{X}\right)\right) \leq \frac{\min \left\{\frac{a_{i}}{c_{i}^{\prime}}\right\}}{1+m \cdot \min \left\{\frac{a_{i}}{c_{i}^{\prime}}\right\}} \leq \frac{a_{i}}{c_{i}^{\prime}} \leq \frac{a_{i}}{c_{i}}<\frac{(n+1) a_{i}}{n c_{i}} .
$$

We note that the replacement of the divisorial part $F$ of $I=\mathcal{O}_{X}(-F) I^{\prime}$ (which makes use of the assumption of the base point freeness of $\left.\left|-K_{X}\right|\right)$ gives the last inequality. Therefore, we have the desired inequality and the proof of Theorem 1.2 is completed by Lemma 2.9.

Now we have completed the proof of Theorem 1.2. Theorem 1.1 follows immediately from Theorem 1.2 since log maximal singularity freeness implies maximal singularity freeness.

Remark 4.1. A difficulty arises when we try to strengthen Theorems 1.1 and 1.2 to K-stability. The point is that a flag ideal $\mathcal{J}=I_{0}+I_{1}+$ $\cdots I_{N-1} t^{N-1}+\left(t^{N}\right)$ of length $N>1$ should satisfy a condition that 
$I_{i} \subset I_{j}$ for any $i<j$, which makes it hard to deform it to an another flag ideal which satisfies Condition 2.10.

Remark 4.2. We explain a generalization of the main theorems. Let $X$ be a Fano manifold and $G \subset \operatorname{Aut}(X)$ a finite subgroup. One can define G-birational superrigidity (see [CS08, Definition 1.30] for the definition). On the other hand, one can also define $G$-equivariant slope stability as the condition that $\mathrm{DF}(\mathcal{B}, \mathcal{L}(-E))>0$ for every deformation to the normal cone derived from the ideal $\mathcal{J}=I+(t) \subset \mathcal{O}_{X \times \mathbb{A}^{1}}$, where $I$ is a $G$-invariant ideal of $\mathcal{O}_{X}$ (cf. [OS12, Section 2.2]). Note that when $G=\{\mathrm{id}\}$ is the trivial group the $G$-birational superrigidity (resp., $G$ equivariant slope stability) coincides with usual birational superrigidity (resp., slope stability).

We can prove the following result with minor natural modifications. Let $X$ be a Fano manifold. Assume that there is a finite subgroup $G \subset \operatorname{Aut}(X)$ with the following properties.

(i) $X$ is $G$-birationally superrigid.

(ii) For every $G$-invariant nonzero effective divisor $D$ on $X$, one has $D \geq-K_{X}$

(iii) Every $G$-invariant nonzero effective divisor $D$ on $X$ is $G$-base point free.

Then $X$ is $G$-equivariantly slope stable.

Here, an effective divisor $D$ on $X$ is $G$-base point free if for any point $x \in X$ there is a $G$-invariant effective divisor $D^{\prime} \in|D|$ which does not contain $x$.

We explain how to modify our arguments in order to prove the above generalization. It is enough to prove the inequality (2) assuming that $I \subset \mathcal{O}_{X}$ is $G$-invariant. By the condition (iii) above, we can deform $I$ keeping the $G$-invariance so that Condition 2.10 holds, which we assume without loss of generality from now on. In order to prove inequality (2) for exceptional divisors with divisorial centers, it is enough to prove the inequality $\operatorname{Sesh}\left(I,\left(X,-K_{X}\right)\right) \leq 1 / d_{i}$ (see Section 3 for $\left.d_{i}\right)$, which follows from condition (ii) above. In order to prove inequality (2) for exceptional divisors with higher codimensional centers, it is enough to $\operatorname{show}$ that $\operatorname{lct}(X, \Sigma) \geq 1 / l$, where $\Sigma:=\Sigma_{I^{\prime}, l}^{(c)} \subset\left|-l K_{X}\right|$ is a $G$ invariant movable linear system constructed as in Section 4 . It follows from condition (i) above and [CS08, Theorem 1.31] that $\operatorname{lct}(X, \Sigma) \geq$ $\operatorname{ct}(X, \Sigma) \geq 1 / l$, which completes the proof. 


\section{A CONJECTURE}

Note that we treat in this paper a special class of Fano manifolds of Picard rank 1 (i.e., $\operatorname{Pic}(X) \cong \mathbb{Z}$ ). More generally, we expect the following.

Conjecture 5.1. For an arbitrary Fano manifold $X$ of Picard rank 1, $\left(X,-K_{X}\right)$ is K-semistable.

We note some supporting evidences here. First, it is proved in F13] that $\left(X,-K_{X}\right)$ is slope stable with respect to divisors. Recall also that we proved a stronger statement in section 3 under the additional assumption of Fano index 1. Moreover, the results of Hwang, Kim, Lee, Park [HKLP11, Theorem1.3] and Fujita [F11, Theorem 1.1], also show slope semistablity along smooth curves.

We also remark that we cannot expect strict (poly)stability as, for instance, small deformations of the Mukai-Umemura 3-fold are not Kpolystable but K-semistable (see [Tia97]).

\section{REFERENCES}

[CT08] X. X. Chen and G. Tian, Geometry of Kähler metrics and foliations by holomorphic discs, Publ. Math. Inst. Hautes Études Sci. vol. 107 (2008), 1-107.

[C03] I. A. Cheltsov, Nonrationality of a four-dimensional smooth complete intersection of a quadric and a quadric not containing a plane, Mat. Sb. vol. 194 (2003), 95-116; translation in Sb. Math. vol. 194 (2003), 1679-1699.

[C04] I. A. Cheltsov, Birationally super-rigid cyclic triple spaces, Izv. Ross. Akd. Nauk Ser. Mat. vol. 6 (2004), 169-220; translation in Izv. Math. 68 (2004), 1229-1275.

[C05] I. A. Cheltsov, Birationally rigid Fano varieties, Uspekhi Mat. Nauk vol. 60 (2005), 71-160; translation in Russian Math. Surveys vol. 60, $875-965$.

[CS08] I. A. Cheltsov and K. A. Shramov, Log canonical thresholds of smooth Fano threefolds (with an appendix by Jean-Pierre Demailly), Uspekhi Mat. Nauk. 63 (2008), no. 5, 73-180; translation in Russian Math. Surveys 63 (2008), no. 5, 859-958.

[CDS12] X. Chen, S. Donaldson, S. Sun, Kähler-Einstein metrics and stability, arXiv:1210.7494. (2012)

[Co95] A. Corti, Factoring birational maps of 3-folds after Sarkisov, J. Alg. Geom. vol. 4 (1995), 223-254.

[Don02] S. K. Donaldson, Scalar curvature and stability of toric varieties, J. Differential Geom. vol. 62, (2002) 289-349.

[Don05] S. K. Donaldson, Lower bounds on the Calabi functional, J. Differential Geom. vol. 70, (2005) 453-472.

[dF13] T. de Fernex, Birationally rigid hypersurfaces, to appear in Invent. Math. 
[F11] K. Fujita, Fano manifolds which are not slope stable along curves, Proc. Japan Acad. Ser. A Math. Sci. 87 (2011), no. 10, 199-202.

[F13] K. Fujita, Towards a criterion for slope stability of Fano manifolds along divisors, arXiv:1301.4538, (2013).

[HKLP11] J-M. Hwang, H. Kim, Y. Lee and J. Park, Slope of smooth rational curves in a Fano manifold, Bull. London Math. Soc. (2011).

[I79] V. A. Iskovskikh, Birational automorphisms of three-dimensional algebraic varieties, Current problems in mathematics vol. 12 (Russian), VINITI, Moscow (1996), 159-236; translation in J. Soviet Math. vol. 13 (1980), 815-868.

[IM71] V. A. Iskovskih, Yu. I. Manin, Three dimensional quartics and counterexamples to the Lüroth problem, Math. USSR. Sb. vol. 82 (1996), $3528-3613$.

$[\mathrm{K}+92] \quad J$. Kollár, et al, Flips and abundance for algebraic threefolds, Astérisque 211, Soc. Math. de France, 1992.

[Li13] C. Li, Yau-Tian-Donaldson correspondence for K-semistable Fano manifolds, arXiv:1302.6681 (2013).

[LX11] C. Li, C. Xu, Special test configurations and K-stability of $\mathbb{Q}$-Fano varieties, arXiv:1111.5398.

[Mum77] D. Mumford, Stability of Projective Varieties, Enseignement Math. vol. 23 (1977).

[Od13a] Y. Odaka, A generalization of the Ross-Thomas slope theory, to appear in Osaka J. Math. vol. 50 no. 1 (2013).

[Od13b] Y. Odaka, On parametrization, optimization and triviality of test configurations, to appear in Proceedings of A.M.S.

[OS12] Y. Odaka, Y. Sano, Alpha invariants and K-stability of $\mathbb{Q}$-Fano variety, Advances in Mathematics 229 (2012), no. 5, 2818-2834.

[P97] A. V. Pukhlikov, Birational automorphisms of double spaces with singularities, J. Math. Sci. (New York) vol. 85 (1997), 2128-2141.

[P98] A. V. Pukhlikov, Birational automorphisms of Fano hypersurfaces, Invent. Math. vol. 134 (1998), 401-426.

[P00] A. V. Pukhlikov, Birationally rigid double Fano hypersurfaces, Mat. Sb. vol. 191 (2000), 101-126; translation in Sb. Math. 191 (2000), 883-908.

[P01] A. V. Pukhlikov, Birationally rigid Fano complete intersections, J. Reine Angew. Math. vol. 541 (2001), 55-79.

[P03] A. V. Pukhlikov, Birationally rigid iterated Fano double covers, Izv. Ross. Akad. Nauk Ser. Mat. 67 (2003), no. 3, 139-182; translation in Izv. Math. 67 (2003), no. 3, 555-596.

[P07] A. V. Pukhlikov, Birationally rigid varieties. I. Fano varieties, Uspekhi Mat. Nauk 62 (2007), no. 5, 15-106 Russian Math. Surveys 62 (2007), no. 5, 857-942.

[P09] A. V. Pukhlikov, Birational geometry of algebraic varieties with a pencil of Fano cyclic covers, Pure Appl. Math. Q. 5 (2009), no. 2, 641-700.

[RT07] J. Ross and R.P. Thomas, A study of the Hilbert-Mumford criterion for the stability of projective varieties, J. Algebraic Geom. vol. 16, (2007) 201-205.

[Stp08] J. Stoppa, K-stability of constant scalar curvature Kähler manifolds, Advances in Mathematics, 221 (2009), 1397-1408. 
[Tia87] G. Tian, On Kähler-Einstein metrics on certain Kähler manifolds with $C_{1}(M)>0$, Invent. Math. vol. 89, (1987) 225-246.

[Tia97] G. Tian, Kähler-Einstein metrics with positive scalar curvature, Invent. Math. vol. 130, (1997) 1-37.

[Tia12] G. Tian, K-stability and Kähler-Einstein metrics, arXiv:1212.4669. (2012)

Department of Mathematics, Faculty of Science, Kyoto University

E-mail address: yodaka@math.kyoto-u.ac.jp

Department of Mathematics, Faculty of Science and Engineering, SAGA UNIVERSITY

E-mail address: okada@cc.saga-u.ac.jp 